"This document is the Accepted Manuscript version of a Published Work that appeared in final form in The Journal of Physical Chemistry A, copyright (C) American Chemical Society after peer review and technical editing by the publisher. To access the final edited and published work see https://pubs-acs-org.uml.idm.oclc.org/doi/10.1021/jp406821g”.

\title{
Rotational Spectra and Structures of the van der Waals Dimers of Argon with 2- Fluoropyridine and 3-Fluoropyridine
}

\author{
Ming Sun, Mahdi Kamaee and Jennifer van Wijngaarden* \\ Department of Chemistry, University of Manitoba, Winnipeg Manitoba, R3T 2N2 \\ Canada
}

*Corresponding author

Email: vanwijng@cc.umanitoba.ca

Phone: (204)474-8379

Fax: (204)474-7608 


\begin{abstract}
The ground state rotational spectra of the van der Waals dimers of 2-fluoropyridine and 3-fluoropyridine with argon have been investigated using both Fourier transform microwave (FTMW) and chirped pulse Fourier transform microwave (cp-FTMW) spectroscopies between 4 and $26 \mathrm{GHz}$. The rotational constants and ${ }^{14} \mathrm{~N}$ nuclear quadrupole coupling constants derived from fitting the observed transitions were used to estimate the van der Waals coordinates of the two complexes. These are in good agreement with the lowest energy structures determined via MP2 calculations (6$311++\mathrm{G}(2 \mathrm{~d}, 2 \mathrm{p}))$ and have the Ar atom sitting above the plane of the aromatic ring. The center of mass separation of the two moieties in the dimers is between 3.5 and $3.6 \AA$ and the Ar atom lies $5^{\circ}$ to $11^{\circ}$ off the monomer $\mathrm{c}^{\prime}$-axis toward the nitrogen atom of the pyridine backbone.
\end{abstract}

KEYWORDS: rotational spectroscopy; van der Waals complex; aromatic ring; pyridine; supersonic jet. 


\section{INTRODUCTION}

From the time of the development of Balle-Flygare-type Fourier transform microwave (FTMW) spectroscopy in the late $1970 \mathrm{~s},{ }^{1,2,3}$ this technique has proven to be a powerful method for observing the rotational spectra of weakly bound molecular complexes in the gas phase due to its high resolution and sensitivity. ${ }^{2}$ With significant advances in high-speed digital electronics during the next decades, the development of modern chirped-pulse Fourier transform microwave (cp-FTMW) spectroscopy ${ }^{4}$ provided a more efficient way to examine weakly bound molecular complexes due to its broadband nature. ${ }^{5,6,7}$ In this paper, we combine both cp-FTMW and Balle-Flygare-type FTMW techniques to examine the argon complexes of two monofluoropyridines: 2fluoropyridine and 3-fluoropyridine. The molecular monomers were well-characterized in our recent work using the same techniques. ${ }^{8}$

Rare gases, such as $\mathrm{Ar}$ and $\mathrm{Ne}$, were previously shown to form two-body (or even larger) weakly bound complexes with five-membered or six-membered aromatic ring molecules. ${ }^{7,9,10}$ From the center of mass $(\mathrm{CM})$ of the molecular monomer, the rare gas atom normally resides more than $3 \AA$ above the ring (which is much longer than any intramolecular bond length) and lies almost perpendicular to the ring plane. For monomers such as furan or pyridine with a symmetry plane perpendicular to the ring plane, the complexes are always found to have the equilibrium position of the rare gas atom in that symmetry plane. To understand the internal dynamics of such van der Waals complexes, a pseudodiatomic model is normally adopted for the dimers by assuming a

rigid aromatic monomer with large amplitude vibrations around the rare gas atom. ${ }^{2,7,9,10}$ Based on that model, (1) a zero-point vibrationally-averaged structure can be derived 
from the experimental rotational constants of both the monomer and the complex; (2) the depth of the pseudodiatomic potential well can be estimated from the centrifugal distortion constants. For a simple monomer bearing atoms with electric quadrupole moments, the experimentally-determined nuclear quadrupole coupling constants from both the monomer and complex can provide an alternative way to analyze the vibrationally-averaged structure of the complex. ${ }^{2,11}$ For the pyridine-Ar complex, the coupling-constant-determined structure and the rotational-constant-determined structure are roughly consistent as the average angular displacements of the Ar atom around the a' and $b^{\prime}$ principal inertial axes of the monomer differed by only about $2^{\circ} .{ }^{9,10}$ In this work, we present ab initio calculations as well as the results of our recent microwave spectroscopic measurements of the Ar complexes with 2-fluoropyridine and 3fluoropyridine. By analyzing the spectra of the complexes, we estimate their structures and discuss the implications of this work in relation to studies of other weakly bound complexes with aromatic ring molecules.

\section{EXPERIMENT}

The pure rotational spectra of the Ar complexes with 2- and 3-fluoropyridine were first observed using our broadband chirped pulse Fourier transform microwave (cpFTMW) instrument which has been previously described. ${ }^{12}$ Survey spectra were typically measured in $2 \mathrm{GHz}$ windows between $8-14 \mathrm{GHz}$ and the observed transitions had linewidths of $\sim 200 \mathrm{kHz}$ FWHM when individual FIDs (free induction decays) were acquired for $20 \mu$ s. Samples of each compound were prepared as a gas mixture of 0.04$0.08 \%$ fluoropyridine in argon and delivered to the pulsed nozzle with a backing pressure 
of $50 \mathrm{psi}(\sim 350 \mathrm{kPa})$. These initial survey spectra guided our experiments using the more sensitive Balle-Flygare FTMW spectrometer ${ }^{13}$ which was used to record individual hyperfine components with higher resolution $(\sim 7 \mathrm{kHz}$ FWHM full width at half maximum) and to seek weaker transitions from 4-26 GHz. Gas mixtures for the FTMW measurements were more dilute $(0.03 \%)$ and maintained at a higher backing pressure (80 psi or $550 \mathrm{kPa}$ ) to optimize the signal.

\section{THEORETICAL CALCULATIONS}

The minimum energy geometries of the complexes were estimated at the MP2 level $(6-311++\mathrm{g}(2 \mathrm{~d}, 2 \mathrm{p}))$ using the Gaussian09W software package. ${ }^{14}$ The two monomer geometries were taken from previous $a b$ initio calculations and were assumed to be unchanged upon complexation with Ar. A series of counterpoise-corrected single point energy calculations was done for each complex in which it was assumed that the Ar atom lies above the plane of the ring. This was consistent with the earlier studies of the Arpyridine complex ${ }^{9,10}$ and with the observed cp-FTMW spectrum. Three parameters were used to describe the orientation of Ar above the ring: $\mathrm{R}$ (the distance between the Ar atom and the center of mass (CM) of the monomer ring), $\theta$ (the Ar-CM-Nitrogen angle) and $\phi$ (the angle between the plane of the ring and the plane containing Ar-CM-N). To narrow down the range of geometric parameters, a rough scan was initially done using increments of $0.1 \AA$ in $\mathrm{R}$ and $5^{\circ}$ in the two angles. A finer scan in steps of $0.01 \AA$ in $\mathrm{R}$ and $1^{\circ}$ in the two angles was subsequently performed. The minimum energy geometries are schematically shown in Figure 1 looking down on the monomer a'b'-planes (where the prime denotes the monomer axes) and in Figure 2 looking down on the dimer ab-planes. 
These correspond to values of $\mathrm{R}, \theta, \phi$ of $\left(3.54 \AA, 88^{\circ}, 85^{\circ}\right)$ and $\left(3.58 \AA, 82^{\circ}, 86^{\circ}\right)$ for the 2-fluoro and 3-fluoropyridine complexes with Ar, respectively. Similar calculations for pyridine yielded values of $\left(3.55 \AA, 86^{\circ}, 90^{\circ}\right)$.

\section{RESULTS AND ANALYSIS}

In our original investigation of the 2-fluoropyridine and 3-fluoropyridine monomers, a few dozen weak transitions were observed in the cp-FTMW spectra which could not be attributed to the parent rings or their ${ }^{13} \mathrm{C}$ analogs. Upon repeating the data acquisition with $\mathrm{Ne}$ as a carrier gas, these weak transitions were not found. These features were susbsequently assigned to the van der Waals dimers of the two rings with Ar. Using the cp-FTMW spectrum of each as a guide, we measured the ${ }^{14} \mathrm{~N}$ hyperfine splitting of these and other transitions of the normal species using our Balle-Flygare FTMW instrument. Transitions due to the ${ }^{13} \mathrm{C}$ isotopologues were not observed in the cp-FTMW spectra and were not sought in the subsequent higher resolution experiments as the observed intensities of the parent species were fairly unstable. The issue of pyridine contamination or breakdown was first realized during our earlier study of the monomers and required frequent flushing of the gas mixing manifold to maintain suitable emission signals. Additionally, after working with pyridine samples over several weeks, the diffusion pump oil needed to be replaced to maintain a suitable backing pressure for the jet expansion.

\section{i) 2-fluoropyridine}

Based on Stark measurements, the 2-fluoropyridine monomer has significant dipole components along the $\mathrm{a}^{\prime}$ - and $\mathrm{b}^{\prime}$ - principal inertial axes $\left(\mu_{\mathrm{a}}=2.8 \mathrm{D}\right.$ and $\mu_{\mathrm{b}}=1.9$ 
D) ${ }^{15}$ which give rise to strong $a$-type and $b$-type transitions in the microwave region. In the complex, these roughly correspond to the b-and c- inertial axes, respectively and their dipole components based on the $a b$ initio structure are estimated to be $\mu_{\mathrm{b}}=3.1 \mathrm{D}$ and $\mu_{\mathrm{c}}=2.0 \mathrm{D}$ with a small component along the van der Waals axis, $\mu_{\mathrm{a}}=0.3 \mathrm{D}$. As a result, both $b$-type and $c$-type transitions were sought for the complex and a sample spectrum for 2-fluoropyridine-Ar showing the ${ }^{14} \mathrm{~N}$ nuclear quadrupole hyperfine structure is given in Figure 3.

In total, 360 hyperfine components from 117 different rotational transitions of the parent moiety were measured between 4 and $25 \mathrm{GHz}$. The observed transitions in this region span quantum numbers $J^{\prime}=2$ through $J^{\prime}=8$. The ${ }^{14} \mathrm{~N}$ hyperfine constants of 2 fluoropyridine-Ar were calculated in G09 at the minimum energy geometry using the keyword Pickett and the off-diagonal nuclear quadrupole coupling constants were found to be relatively large although the observed transitions were not particularly sensitive to these parameters. The spectra were fit in Pickett's SPFIT program ${ }^{16}$ using Watson's Areduced Hamiltonian ( $I^{r}$ representation) with the off-diagonal components of the nuclear quadrupole coupling tensor fixed at the ab initio values. The spectroscopic constants are reported in Table 1 including standard errors from Kisiel's PIFORM program. ${ }^{17}$ The observed transitions frequencies are provided as supplemental data.

\section{ii) 3-fluoropyridine}

For the 3-fluoropyridine monomer, the two in-plane dipole components are $\mu_{\mathrm{a}}=$ $0.8 \mathrm{D}$ and $\mu_{\mathrm{b}}=2.2 \mathrm{D}$ and both $a$-type and $b$-type transitions were observed..$^{18}$ Compared with its structural isomer, the center of mass (shown in Figure 1) of the ring is quite different due to the position of the heavy fluorine atom although the a'-axis follows the 
C-F bond in both. In the van der Waals dimer, the two axes in the plane of the ring approximately correspond to the b- and c-axes and their dipole components are estimated to be $\mu_{\mathrm{b}}=0.7 \mathrm{D}$ and $\mu_{\mathrm{c}}=2.1 \mathrm{D}$ via computation and that along the a-axis, $\mu_{\mathrm{a}}=0.02 \mathrm{D}$ is negligible. Based on this, both $b$-type and $c$-type transitions were sought for the 3fluoropyridine complex with Ar.

The assignment and analysis of the spectra followed that described above for 2 fluoropyridine-Ar. A total of 244 hyperfine components due to $79 b$-type and $c$-type rotational transitions were measured between 5 and $24 \mathrm{GHz}$. The observed transitions in this region span quantum numbers $J^{\prime}=2$ through $J^{\prime}=9$. As in the previous case, the offdiagonal elements of the nuclear quadrupole coupling tensor were held fixed to the values estimated from $a b$ initio calculations. The spectroscopic constants obtained using Watson's A-reduced Hamiltonian ( $I^{r}$ representation) are provided in Table 1 and the observed transitions frequencies are provided as supplemental data.

\section{DISCUSSION}

In order to estimate the experimental molecular structure of the weakly bound Arpyridine dimer without isotopic substitution, different methods can be used if the geometry of the fluoropyridine ring is assumed to be unaffected upon complexation. One of those methods is called the "extreme Kraitchman" analysis. ${ }^{11,19}$ For this analysis, the Ar complex can be taken as the special isotopologue of the parent fluoropyridine ring with a dummy atom at the Ar position. By using the nonplanar asymmetric Kraitchman equations, one can determine the absolute coordinate values in the fluoropyridine principal inertial axis frame $\left(\mathrm{a}^{\prime}, \mathrm{b}^{\prime}, \mathrm{c}^{\prime}\right)$ as shown below in Equation $1::^{20}$ 
$a^{\prime}=\left[\frac{\Delta P_{a}}{\mu}\left(1+\frac{\Delta P_{b}}{I_{a}^{\prime}-I_{b}^{\prime}}\right)\left(1+\frac{\Delta P_{c}}{I_{a}^{\prime}-I_{c}^{\prime}}\right)\right]^{1 / 2}$ with $\mathrm{a}^{\prime}, \mathrm{b}^{\prime}, \mathrm{c}^{\prime}$ cyclic permutations

where $\mu$ is the reduced mass of the fluoropyridine parent and the Ar atom; $\Delta P_{a}=\frac{1}{2}\left[-\Delta I_{a}+\Delta I_{b}+\Delta I_{c}\right] ; \Delta I_{a}=I_{b}-I_{a}^{\prime}, \Delta I_{b}=I_{c}-I_{b}^{\prime}$ and $\Delta I_{c}=I_{a}-I_{c}^{\prime}$ due to the axis switching from the fluoropyridine principal axis frame $\left(a^{\prime}, b^{\prime}, c^{\prime}\right)$ to the dimer principal axis frame $(\mathrm{a}, \mathrm{b}, \mathrm{c})$ after complexation. To apply this method, Kisiel's program KRA can be used to give absolute coordinates. ${ }^{17}$

An alternative method is to use Kisiel's program RGDFIT $^{17,21,22}$ based on a method first proposed by Suenram et $a .^{21}$ to determine the structure of the Ar-formamide complex. In this program, the three coordinates of the rare gas atom are fitted to the three rotational constants of the complex using a least squares routine. In the absence of spectra corresponding to other isotopologues, as in this case, the structure determined via "extreme Kraitchmann" analysis and the method in the RGFIT program are exactly the same.

Using the ab initio structures as a guide, we matched one set of the coordinates from the RGDFIT analysis to each of our fluoropyridine-Ar complexes. The final results are shown in Table 2 along with the structural parameters of pyridine-Ar and furan-Ar for comparison. ${ }^{10}$ Although only one bond length and two angles are needed to specify the geometry, we provide an additional angle for ease of comparison. The coordinates reported $(\mathrm{R}, \gamma, \theta, \phi)$ are described pictorially in Figure 4 such that $\mathrm{R}$ is the distance from Ar to the center of mass $(\mathrm{CM})$ of the monomer ring, $\gamma$ is the angle between the $\mathrm{c}^{\prime}$-axis of the monomer and the Ar-CM line, $\theta$ is the Ar-CM-N angle and $\phi$ is the angle between the 
Ar-CM-N plane and the CM-N-C5 plane. Based on the results in Table 2, one finds that on complexation, the Ar atom is located above the aromatic rings with the Ar-CM line lying almost perpendicular to the ring plane ( $\gamma$ ranging from $3^{\circ}$ to $11^{\circ}$ ), but tilts slightly towards the heteroatom (the angle $\theta, \angle \mathrm{Ar}-\mathrm{CM}-\mathrm{N}$ or $\angle \mathrm{Ar}-\mathrm{CM}-\mathrm{O}$, ranging from $79^{\circ}$ to $88^{\circ}$ ) in all cases.

In Table 2, the $a b$ initio results for the 2-fluoropyridine-Ar dimer and 3fluoropyridine-Ar dimer in terms of the same coordinates are also listed. In general, the structural parameters from both the ab initio calculation and inertial analysis are roughly consistent with only a few degrees difference in the van der Waals angles and a few hundredths of an Angstrom in the van der Waals bond length. As suggested by Suenram et al., ${ }^{21}$ such discrepancies may be a result of large amplitude zero-point motions which are not accounted for in the $a b$ initio estimates of the equilibrium geometry. Upon complexation, the three translational degrees of freedom of the Ar atom are transformed into three van der Waals vibrational modes, the two bending modes around the monomer principal axes $\mathrm{a}^{\prime}$ and $\mathrm{b}^{\prime}$, as well as the pseudodiatomic stretching mode between the CM of the monomer and the Ar atom. Since the RGFIT program fits three van der Waals coordinates to the three rotational constants of the dimer, the reliability of the results (and the contribution from large amplitude motions) is difficult to gauge.

An alternative way to evaluate the structure of the van der Waals complexes is to use the ${ }^{14} \mathrm{~N}$ quadrupole coupling constants as shown earlier for molecules such as Arpyridine, ${ }^{9,10}$ Ar-trifluoroacetonitrile, ${ }^{11}$ Ar-formamide ${ }^{21}$ and Ar-methyl isocyanide. ${ }^{23}$ For weakly bound complexes with rare gas atoms, the electric field gradients in the monomer should not be affected significantly by the complexation. If the transformations of the ${ }^{14} \mathrm{~N}$ 
quadrupole coupling constants from the inertial axes to the principal quadrupole tensor axes are known for both the monomer and the dimer (and they can be diagonalized), one can use the direction cosines to deduce the rotation matrix between the monomer principal inertial frame $\left(a^{\prime}, b^{\prime}, c^{\prime}\right)$ and the dimer principal inertial frame $(a, b, c)$. By using the derived rotation matrices, along with the Ar-CM distance obtained from the RGDFIT analysis and the $\mathrm{r}_{0}$ structure of the monomer, ${ }^{8}$ we obtained a new estimate of the structural parameters for the two dimers which are included in Table 2. Compared to the inertial analysis, the structure determined via the ${ }^{14} \mathrm{~N}$ hyperfine analysis is in better agreement with the MP2 calculation in both cases. This is likely a result of using ab initio data for the off-diagonal quadrupole coupling constants (as described in Section IV above) when deriving the rotation matrix as these have a strong influence on the result.

The experimental and theoretical results can also be compared using the ${ }^{14} \mathrm{~N}$ quadrupole coupling constants directly. The experimentally-determined constants are shown in Table 3 for the two monomers ${ }^{8}$ as well as their van der Waals complexes with Ar. Using the results from the RGDFIT analysis, the monomer quadrupole tensor of each can be rotated into the principal inertial axis system of the corresponding dimer to obtain an estimate of the quadrupole coupling constants based on experimental results. These are provided in Table 3 for both 2 -fluoropyridine and 3 -fluoropyridine. These show reasonable agreement in terms of size and magnitude with those determined from MP2 calculations which are provided for comparison. The biggest discrepancies again occur for the off-diagonal terms which were not well-determined from the microwave data. 
When the components of the dimers are treated as two separate subunits, the pseudodiatomic stretching mode can be evaluated by the dimer's centrifugal distortion constant $\Delta_{\mathrm{J}}$, which is related to the stretching force constant $\mathrm{k}_{\mathrm{s}}$ by Equation $2:{ }^{24}$

$$
k_{s}=\frac{16 \pi^{4} \mu^{2} R^{2}}{h \Delta_{J}}\left[\left(B^{2}+C^{2}\right)^{2}+2\left(B^{4}+C^{4}\right)\right]
$$

where $\mu$ is the reduced mass of the dimer when treated as two pseudodiatomic subunits; $R$ $\left(\mathrm{R}_{0}\right.$ in Table 5$)$ is the distance from the $\mathrm{CM}$ of the monomer to the Ar atom determined by the inertial analysis; $\mathrm{B}$ and $\mathrm{C}$ are the rotational constants of the dimers. This assumes that the $\mathrm{R}_{0}$ axis of the complex is nearly aligned with the a-axis of the dimer which is true in cases where $\gamma$ is small. With the force constant $k_{\mathrm{s}}$ and the pseudodiatomic distance $R$, the pseudodiatomic stretching frequency as well as other Lennard-Jones potential parameters can be evaluated ${ }^{2,24}$ as listed in Table 4 . In the same table, the potential parameters of pyridine-Ar and furan-Ar are also listed for comparison. ${ }^{10,24}$

With fluorine substitution, the stretching mode becomes weaker with smaller force constants $k_{\mathrm{s}}$, lower stretching frequencies $v_{\mathrm{s}}$, and a more shallow well depth $C$ than in the unsubstituted pyridine-Ar dimer. Compared to pyridine-Ar and 2-fluoropyridine-Ar, the stretching mode in 3-fluoropyridine-Ar dimer is lower frequency with a smaller well depth (3-fluoropyridine-Ar: $173 \mathrm{~cm}^{-1}$; 2-fluoropyridine-Ar: $215 \mathrm{~cm}^{-1}$; pyridine-Ar: 236 $\mathrm{cm}^{-1}$ ). This suggests that the interaction is stronger between 2-fluoropyridine and Ar than in the other complexes and is consistent with the ab initio structures in that the Ar atom sits closer to both the nitrogen and the fluorine atoms (3.70 $\AA$ and $4.29 \AA$, respectively) in 2-fluoropyridine-Ar in comparison to the 3-fluoropyridine-Ar species (3.74 $\AA$ and $4.47 \AA$, respectively). This is due to differences in the electronic structure of the two monomers moieties themselves. The electrostatic potential energy surfaces and the results of the ${ }^{14} \mathrm{~N}$ 
hyperfine analysis for 2-fluoro and 3-fluoropyridine in reference 8 showed an increase in negative charge on the nitrogen atom in the former monomer in comparison to the latter. This was attributed to a hyperconjugation effect. The lone pair of electrons from fluorine can contribute to the $\pi$-bonding system of the ring and the hyperconjugated resonance structure that is most stable for 2-fluoropyridine is that with the negative charge on nitrogen. In 3-fluoropyridine, two hyperconjugated resonance structures have similar energy and place the additional negative charge on the carbons adjacent to the site of fluorination and thus, the charge is more delocalized. This qualitative comparison of the electronic structure of the monomers is consistent with the assertion that the stronger intermolecular interaction occurs in the case of the 2-fluoropyridine-Ar complex.

\section{Acknowledgements}

Funding for this research was provided by the Natural Sciences and Engineering Research Council (NSERC) of Canada through the Discovery Grant and University Faculty Award programmes.

\section{Supporting information}

The assigned transition frequencies for both complexes are provided as supplementary material. This material is available free of charge via the Internet at http://pubs.acs.org 
Table 1. Spectroscopic Constants of the fluoropyridine-Ar dimers.

\begin{tabular}{|c|c|c|}
\hline & 2-fluoropyridine-Ar & 3-fluoropyridine-Ar \\
\hline \multicolumn{3}{|c|}{ Rotational Constants $/ \mathrm{MHz}$} \\
\hline A & $1873.398581(34)$ & $1884.655237(44)$ \\
\hline $\mathrm{B}$ & $1151.265171(26)$ & $1110.892770(30)$ \\
\hline $\mathrm{C}$ & $936.046138(28)$ & $914.212637(57)$ \\
\hline \multicolumn{3}{|c|}{ Centrifugal Distortion Constants $/ \mathrm{kHz}$} \\
\hline$\Delta_{\mathrm{J}}$ & $2.51368(33)$ & $2.88558(97)$ \\
\hline$\Delta \Delta_{\mathrm{JK}}$ & $15.1931(14)$ & $5.5681(35)$ \\
\hline$\Delta_{\mathrm{K}}$ & $-16.6143(17)$ & $-4.0761(33)$ \\
\hline$\delta_{\mathrm{J}}$ & $0.54825(17)$ & $0.72684(46)$ \\
\hline$\Delta_{\mathrm{K}}$ & $8.0716(17)$ & $5.0534(25)$ \\
\hline \multicolumn{3}{|c|}{${ }^{14} \mathrm{~N}$ Nuclear Quadrupole Coupling Constants /MHz } \\
\hline $1.5 \chi_{\mathrm{aa}}$ & $4.1588(10)$ & $4.5406(13)$ \\
\hline $0.25\left(\chi_{\mathrm{bb}}-\chi_{\mathrm{cc}}\right)$ & $0.63496(25)$ & $0.96361(31)$ \\
\hline$\chi_{\mathrm{ab}} \mathrm{a}^{\mathrm{a}}$ & -0.273 & -1.070 \\
\hline$\chi_{\mathrm{ac}^{\mathrm{a}}}$ & 0.071 & 1.257 \\
\hline$\chi_{\mathrm{bc}^{\mathrm{a}}}$ & 2.848 & 2.494 \\
\hline rms error $/ \mathrm{kHz}$ & 3.5 & 3.8 \\
\hline no. lines & 360 & 244 \\
\hline
\end{tabular}

aThe off-diagonal parameters were fixed to those determined by MP2 calculations (6$311++\mathrm{G}(2 \mathrm{~d}, 2 \mathrm{p}))$. 
Table 2. Structural parameters of aromatic ring-Ar dimers obtained by different methods.

\begin{tabular}{ccccc}
\hline \hline & $\mathrm{R}(\AA)$ & $\gamma\left(^{\circ}\right)$ & $\theta\left(^{\circ}\right)$ & $\phi\left(^{\circ}\right)$ \\
\hline Pyridine-Ar $^{\mathrm{a}}$ & 3.545 & 3.5 & 86.5 & 90.0 \\
Furan-Ar $^{\mathrm{a}}$ & 3.541 & 5.5 & 84.5 & 90.0 \\
& & & & \\
2-Fluoropyridine-Ar & & & & \\
Method I $^{\mathrm{b}}$ & 3.540 & 5 & 88 & 85 \\
Method II $^{\mathrm{c}}$ & 3.522 & 8.0 & 84.1 & 84.6 \\
Method III $^{\mathrm{d}}$ & 3.522 & 5.3 & 88.0 & 85.0 \\
& & & & \\
3-Fluoropyridine-Ar & & & & \\
Method I & 3.580 & 9 & 82 & 86 \\
Method II & 3.564 & 10.9 & 79.2 & 88.5 \\
Method III & 3.564 & 8.5 & 82.2 & 86.6 \\
\hline \hline
\end{tabular}

${ }^{a}$ Ref. 10, by analysis of the moments of inertia (Method II).

${ }^{\mathrm{b}}$ Method I: From MP2 (6-311++g(2d,2p)) calculations.

${ }^{c}$ Method II: From inertial analysis (RGDFIT) with ring structures $\left(\mathrm{r}_{0}\right)$ from Ref. 8.

${ }^{\mathrm{d}}$ Method III: From quadrupole hyperfine analysis with R from RGDFIT and ring structures $\left(\mathrm{r}_{0}\right)$ from Ref. 8.

Table 3. ${ }^{14} \mathrm{~N}$ nuclear quadrupole coupling constants of the fluoropyridines and their Ar dimers in MHz.

\begin{tabular}{ccccccc}
\hline \hline & $\chi_{\mathrm{aa}}$ & $\chi_{\mathrm{bb}}$ & $\chi_{\mathrm{cc}}$ & $\chi_{\mathrm{ab}}$ & $\chi_{\mathrm{ac}}$ & $\chi_{\mathrm{bc}}$ \\
2-Fluoropyridine & -0.100 & -2.736 & 2.836 & 2.760 & 0 & 0 \\
& & & & & & \\
2-Fluoropyridine-Ar & & & & & & \\
$\quad \begin{array}{c}\text { Experiment } \\
\text { Method I }\end{array}$ & 2.773 & -0.116 & -2.656 & -0.273 & 0.071 & 2.848 \\
$\quad 2.789$ & -0.086 & -2.703 & -0.273 & 0.071 & 2.848 \\
Method II $^{\mathrm{d}}$ & 2.762 & -0.220 & -2.542 & -0.026 & 0.474 & 2.845 \\
& & & & & & \\
3-Fluoropyridine & -0.035 & -3.466 & 3.501 & -2.751 & 0 & 0 \\
& & & & & & \\
3-Fluoropyridine-Ar & & & & & & \\
$\quad \begin{array}{c}\text { Experiment } \\
\text { Method I }\end{array}$ & 3.027 & 0.414 & -3.441 & -1.070 & 1.257 & 2.494 \\
$\quad$ Method II & 2.964 & 0.490 & -3.454 & -1.070 & 1.257 & 2.494 \\
\hline \hline
\end{tabular}

\footnotetext{
${ }^{\text {a }}$ Ref. 8 (experiment).

${ }^{\mathrm{b}}$ Off-diagonal terms were fixed to ab initio values during spectral analysis

${ }^{c}$ Method I: From MP2 (6-311++g(2d,2p)) calculations.

${ }^{d}$ Method II: From rotating the monomer quadrupole tensor into the dimer inertial axis system based on the RGDFIT analysis.
} 
Table 4. Lennard-Jones potential parameters obtained by pseudodiatomic approximation. ${ }^{\text {a }}$

\begin{tabular}{cccccccc}
\hline \hline & $R_{0}(\AA)^{\mathrm{d}}$ & $B_{0}(\mathrm{MHz})^{\mathrm{e}}$ & $k_{\mathrm{s}}\left(\mathrm{N} \cdot \mathrm{m}^{-1}\right)$ & $v_{\mathrm{s}}\left(\mathrm{cm}^{-1}\right)$ & $B_{\mathrm{e}}(\mathrm{MHz})^{\mathrm{f}}$ & $R_{\mathrm{e}}(\AA)$ & $\epsilon\left(\mathrm{cm}^{-1}\right)$ \\
Pyridine-Ar $^{\mathrm{b}}$ & 3.545 & 1515.089 & 2.70 & 41.5 & 1546.336 & 3.509 & 232.0 \\
Furan-Ar & 3.541 & 1505.979 & 2.69 & 42.5 & 1538.830 & 3.503 & 236.0 \\
2-Fluoropyr-Ar & 3.522 & 1439.620 & 2.54 & 39.0 & 1473.021 & 3.482 & 215.0 \\
3-Fluoropyr-Ar & 3.564 & 1405.319 & 2.00 & 34.6 & 1441.368 & 3.520 & 172.9 \\
\hline \hline
\end{tabular}

a The method as applied to a pseudodiatomic complex is described in Ref. 2 and 24.

b Ref. 10.

${ }^{c}$ Ref. 24.

d The pseudodiatomic rotational constant based on $\mathrm{R}_{0}$.

e The equilibrium pseudodiatomic rotational constant

${ }^{\mathrm{f}}$ The equilibrium pseudodiatomic bond length based on $\mathrm{R}_{\mathrm{e}}$. 


\section{Figure Captions}

Figure 1: Schematic representations of the structures of the fluoropyridine-Ar complexes looking down on the monomer $a^{\prime} b^{\prime}-$ plane based on MP2 $(6-311++g(2 d, 2 p))$ calculations. The small purple sphere shows the center of mass of the monomer ring.

Figure 2: Schematic representations of the structures of the fluoropyridine-Ar complexes looking down on the dimer ab-plane based on MP2 $(6-311++\mathrm{g}(2 \mathrm{~d}, 2 \mathrm{p}))$ calculations.

Figure 3: Sample Balle-Flygare FTMW spectrum of the $4_{22}-33_{12}$ transition of the 2fluoropyridine-Ar complex showing the hyperfine splitting arising due to the ${ }^{14} \mathrm{~N}$ quadrupole nucleus.

Figure 4: Schematic definitions of the van der Waals coordinates reported for the structures of the dimer complexes in relation to the monomer $a^{\prime}, b^{\prime}, c^{\prime}$-axis system. Not

labelled for simplicity are the coordinates R (Ar-CM distance) and $\phi$ (angle between ArCM-N plane and CM-N-C5 plane). 
Figure 1: Sun et al.

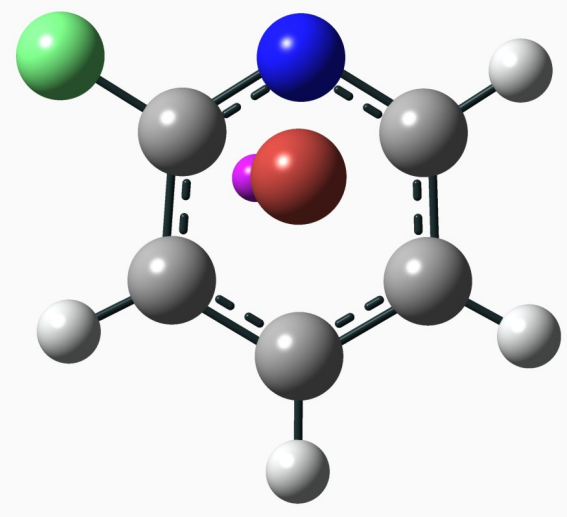

2-fluoropyridine-Ar

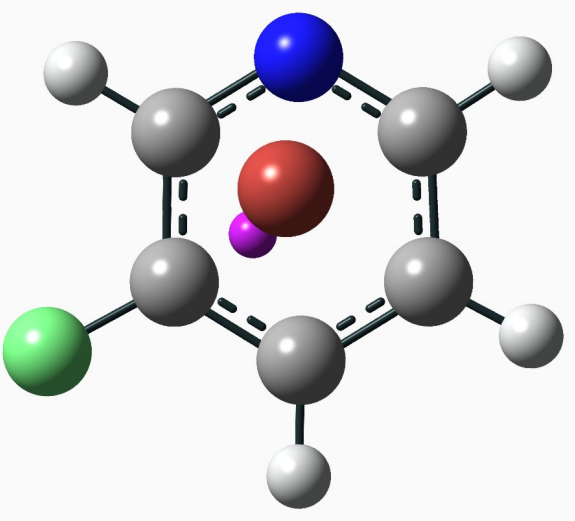

3-fluoropyridine-Ar

Figure 2: Sun et al.

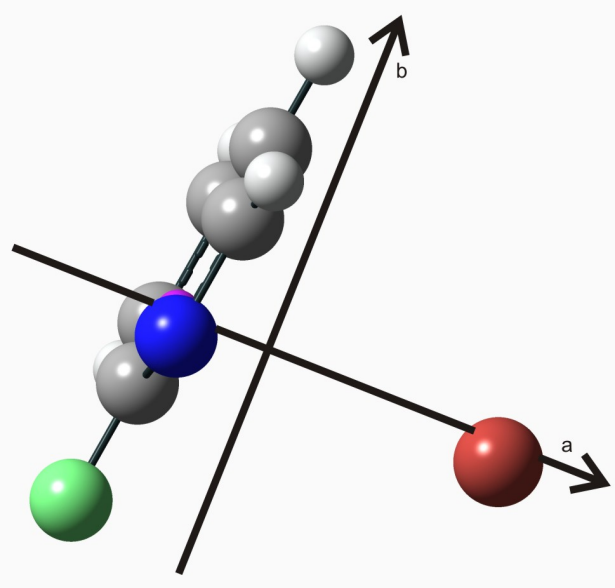

2-fluoropyridine-Ar

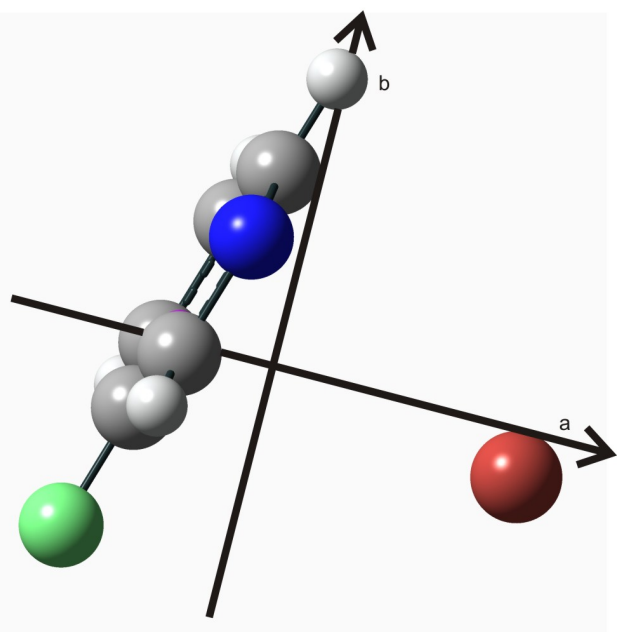

3-fluoropyridine-Ar 
Figure 3: Sun et al.

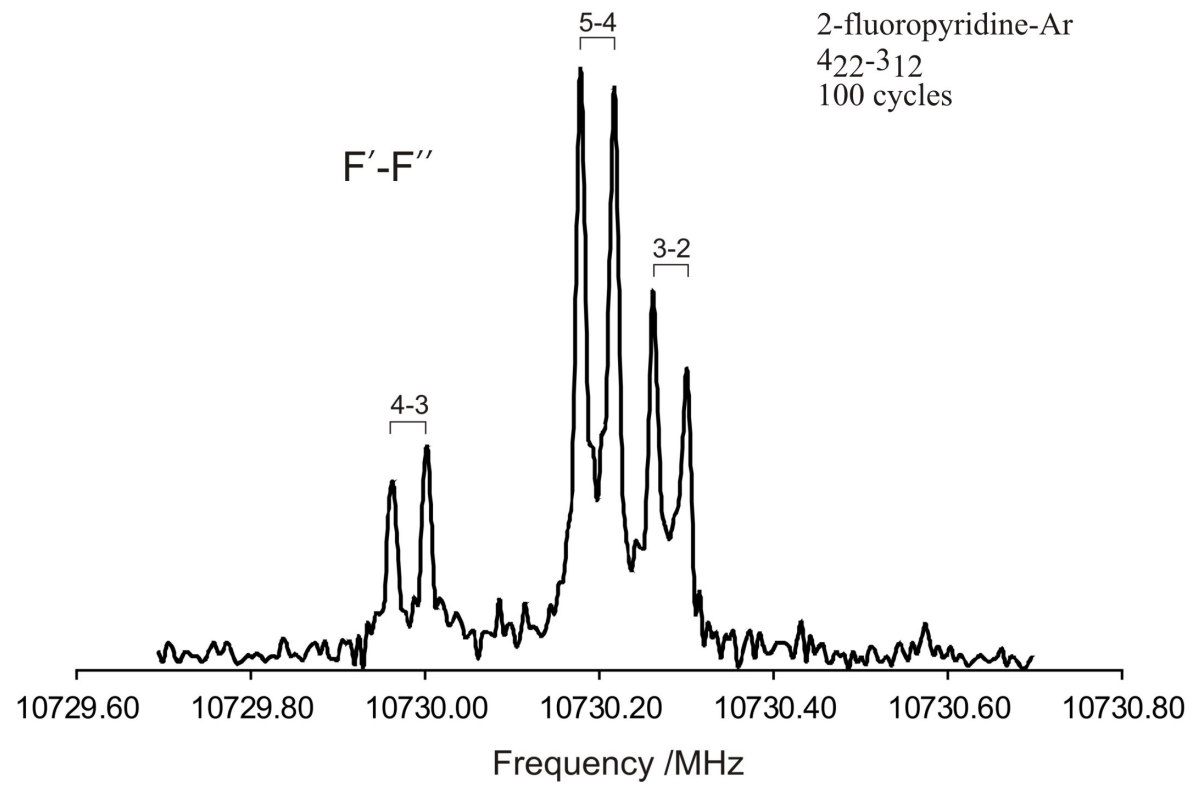

Figure 4: Sun et al.

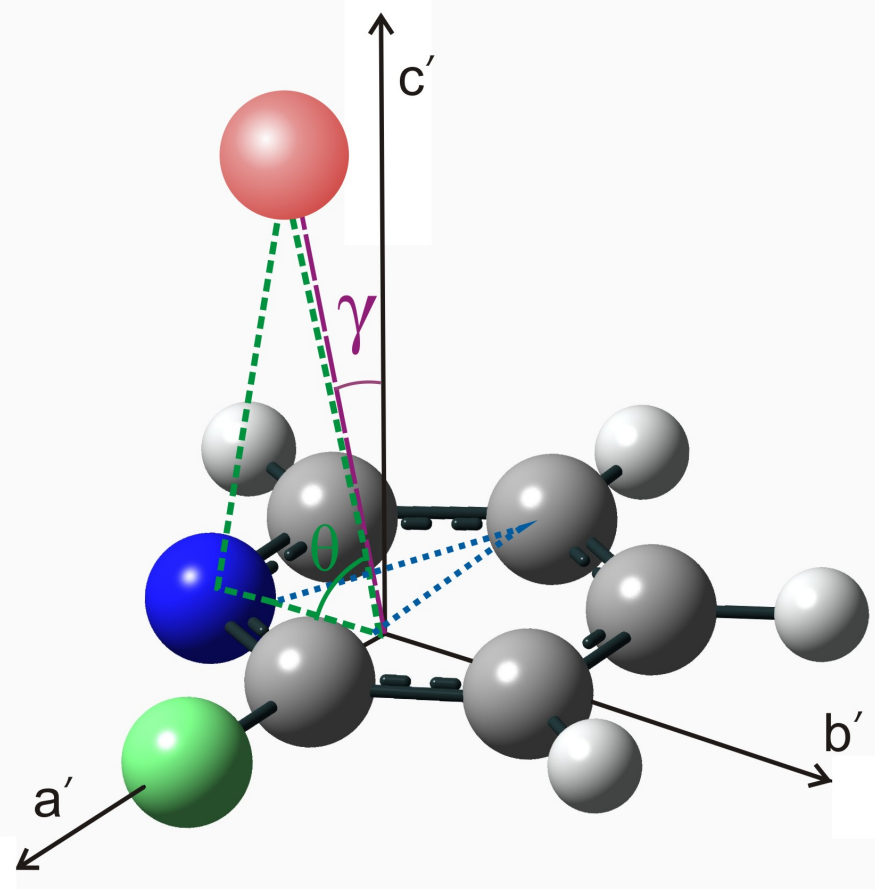




\section{References}

${ }^{1}$ Ekkers, J.; Flygare, W. H. Pulsed Microwave Fourier Transform Spectrometer. Rev. Sci. Instrum. 1976, 47, 448-454.

${ }^{2}$ Balle, T. J.; Campbell, E. J.; Keenan, K. R.; Flygare, W. H. A New Method for Observing the Rotational Spectra of Weak Molecular Complexes: KrHCl. J. Chem. Phys. 1980, 72, 922-932.

${ }^{3}$ Balle, T. J.; Flygare, W. H. Fabry-Perot Cavity Pulsed Fourier Transform Microwave Spectrometer with a Pulsed Nozzle Particle Source. Rev. Sci. Instrum. 1981, 52, 33-45.

${ }^{4}$ Brown, G. G.; Dian, B. C.; Douglass, K. O.; Geyer, S. M.; Shipman, S. T.; Pate, B. H. A Broadband Fourier Transform Microwave Spectrometer Based on Chirped Pulse Excitation. Rev. Sci. Instrum. 2008, 79, 053103.

${ }^{5}$ Pérez, C.; Muckle, M. T.; Zaleski, D. P.; Seifert, N. A.; Temelso, B.; Shields, G. C.; Kisiel, Z.; Pate, B. H. Structures of Cage, Prism, and Book Isomers of Water Hexamer from Broadband Rotational Spectroscopy. Science, 2012, 336, 897-901.

${ }^{6}$ Stephens, S. L.; Walker, N. R.; Legon, A. C. Rotational Spectra and Properties of Complexes B--- $\mathrm{ICF}_{3}(\mathrm{~B}=\mathrm{Kr}$ or $\mathrm{CO})$ and a Comparison of the Efficacy of $\mathrm{ICl}$ and $\mathrm{ICF}_{3}$ as Iodine Donors in Halogen Bond Formation. J. Chem. Phys. 2011, 135, 224309. 
${ }^{7}$ Neill, J. L.; Shipman, S. T.; Alvarez-Valtierra, L.; Lesarri, A.; Kisiel, Z.; Pate, B. H.

Rotational Spectroscopy of Iodobenzene and Iodobenzene-Neon with a Direct Digital 2-8

GHz Chirped-Pulse Fourier Transform Microwave Spectrometer. J. Mol. Spectrosc. 2011, 269, 21-29.

${ }^{8}$ van Dijk, C. W.; Sun, M.; van Wijngaarden, J. Microwave Rotational Spectra and Structures of 2-Fluoropyridine and 3-Fluoropyridine. J. Phys. Chem. A. 2012, 116, 40824088.

${ }^{9}$ Spycher, R. M.; Petitprez, D.; Bettens, F. L.; Bauder, A. Rotational Spectra of Pyridine(Argon) $)_{n}, \mathrm{n}=1,2$, Complexes and their Vibrationaly Averaged Structures. J. Phys. Chem. 1994, 98, 11863-11869.

${ }^{10}$ Klots, T. D.; Emilsson, T.; Ruoff, R. S.; Gutowsky, H. S. Microwave Spectra of Noble Gas-Pyridine Dimers: Argon-Pyridine and Kr-Pyridine. J. Phys. Chem. 1989, 93, 12551261.

${ }^{11}$ Lin, W.; Novick, S. E. The Microwave Spectrum and Structure of the Argon Trifluoroacetonitrile Complex. J. Mol. Spectrosc. 2007, 243, 32-36.

${ }^{12}$ Evangelisti, L.; Sedo, G.; van Wijngaarden, J. Rotational Spectrum of 1,1,1-Trifluoro2-butanone Using Chirped-Pulse Fourier Transform Microwave Spectroscopy. J. Phys. Chem. A. 2011, 115, 685-690. 
${ }^{13}$ Sedo, G.; van Wijngaarden, J. Fourier Transform Microwave Spectra of a "New" Isomer of OCS-CO 2. J. Chem. Phys. 2009, 131, 044303.

${ }^{14}$ Frisch, M. J. et al., Gaussian 09, Revision A.02, Gaussian, Inc., Wallingford CT, 2009.

${ }^{15}$ Sharma, S. D.; Doraiswamy, S.; Legell, H.; Mäder, H.; Sutter, D. Microwave Spectrum, Dipole Moment and Quadrupole Coupling Constants of Orthofluoropyridine. Z. Naturforsch. Teil A. 1971, 26a, 1342-1345.

${ }^{16}$ Pickett, H. M. The Fitting and Prediction of Vibration-Rotation Spectra with Spin Interactions. J. Mol. Spectrosc. 1991, 148, 371-377.

${ }^{17}$ Kisiel, Z. PROSPE - Programs for Rotational SPEctroscopy, $<$ http://info.ifpan.edu.pl/ kisiel/prospe.htm $>$.

${ }^{18}$ Sharma, S. D.; Doraiswamy, S. Microwave Spectrum, Dipole Moment, Quadrupole Coupling Constants, and Centrifugal Distortion Constants of 3-Fluoropyridine. J. Mol. Spectrosc. 1976, 59, 216-225.

${ }^{19}$ Kraitchman, J. Determination of Molecular Structure from Microwave Spectroscopic Data. Am. J. Phys. 1953, 21, 17-24. 
${ }^{20}$ Gordy, W.; Cook, R. L. Microwave Mol. Spectra; Wiley, New York, 1984.

${ }^{21}$ Suenram, R. D.; Fraser, G. T.; Lovas, F. J.; Gillies, C. W.; Zozom, J. Microwave Spectrum, Structure, and Electric Dipole Moment of the Ar-Formamide van der Waals Complex. J. Chem. Phys. 1988, 89, 6141-6146.

${ }^{22}$ Kisiel, Z.; Fowler, P. W.; Legon, A. C. Rotational Spectra and Structures of the van der Waals Dimers of Ar with a Series of Fluorocarbons: Ar... $\mathrm{CH}_{2} \mathrm{CHF}, \mathrm{Ar} . . \mathrm{CH}_{2} \mathrm{CF}_{2}$, and Ar...CHFCF 2 . J. Chem. Phys. 1991, 95, 2283-2291.

${ }^{23}$ Blanco, S.; Lister, D. G.; Legon, A. C.; Rego, C. A. Microwave Spectrum and Geometry of the Methyl Isocyanide-Argon Complex. J. Chem. Soc., Faraday Trans. 1997, 93, 1287-1290.

${ }^{24}$ Kukolich, S. G. Microwave Structure Measurements on the Furan-Argon Complex $J$. Am. Chem. Soc. 1983, 105, 2207-2210. 
TOC image

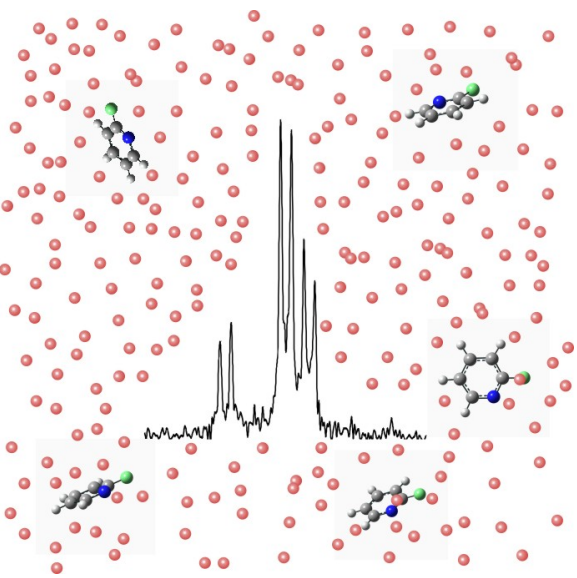

